\title{
Past-A, a Novel Proton-Associated Sugar Transporter, Regulates Glucose Homeostasis in the Brain
}

\author{
Noriaki Shimokawa, ${ }^{1,2,3}$ Junichi Okada, ${ }^{2,3}$ Kaisa Haglund, ${ }^{1}$ Ivan Dikic, ${ }^{1}$ Noriyuki Koibuchi, ${ }^{2,3}$ and \\ Mitsuhiko Miura² \\ ${ }^{1}$ Molecular Signaling Group, Ludwig Institute for Cancer Research, Uppsala, S-75124, Sweden, ${ }^{2}$ Department of \\ Physiology First Division, Gunma University School of Medicine, Maebashi-shi 371-8511, Japan, and ${ }^{3}$ Core Research for \\ Evolutional Science and Technology, Japan Science and Technology Corporation, Kawaguchi, Saitama 332-0012, Japan
}

The ventral medullary surface (VMS) of the medulla oblongata is known to be the site of the central chemosensitive neurons in mammals. These neurons sense excess $\mathrm{H}^{+} / \mathrm{CO}_{2}$ dissolved in the CSF and induce hyperventilation. To elucidate the mechanism of neuronal cell adaptation to changes of $\mathrm{H}^{+} / \mathrm{CO}_{2}$, we screened for hypercapnia-induced genes in the VMS. Here, we report cloning and characterization of a novel gene called proton-associated sugar transporter-A (Past- $A$ ), which is induced in the brain after hypercapnia and mediates glucose uptake along the $\mathrm{pH}$ gradient. Past-A comprises 751 amino acid residues containing 12 membrane-spanning helices, several conserved sugar transport motifs, three proline-rich regions, and leucine repeats. Past- $A$ transcript was expressed predominantly in the brain. Moreover, the Past-Aimmunoreactive neural cells were found in the VMS of the medulla oblongata, and the number of immunoreactive cells was increased by hypercapnic stimulation. Transient transfection of Past- $A$ in COS-7 cells leads to the expression of a membraneassociated $82 \mathrm{kDa}$ protein that possesses a glucose transport activity. The acidification of extracellular medium facilitated glucose uptake, whereas the addition of carbonyl cyanide $m$ chlorophenylhydrazone, a protonophore, inhibited glucose import. Together, our results indicate that Past-A is a brain-specific glucose transporter that may represent an adaptation mechanism regulating sugar homeostasis in neuronal cells after hypercapnia.

Key words: differential display; hypercapnia-induced gene; proton-associated sugar transporter; glucose uptake; ventral medullary surface; glucose homeostasis
The ventilatory response to hypercapnia or acidosis is mediated by central chemoreceptor neurons in the ventrolateral surface of the medulla (Loeschcke, 1982). They are distributed over the ventral medullary surface (VMS) at the rostral medulla, which is bathed in CSF, and are stimulated by excess $\mathrm{H}^{+} / \mathrm{CO}_{2}$ (Schlaefke et al., 1970; Fukuda and Honda, 1975). The cellular properties of these chemosensitive neurons are that they belong to the specific serotonergic subset of neurons, are highly sensitive to small changes in extracellular $\mathrm{pH}(\mathrm{pH}$ between 7.2 and 7.6), and are able to trigger hypercapnic and/or tachypnic response (Richerson et al., 2001). Chemosensitivity of neurons in VMS increases in the postnatal period in rats in parallel with development of respiratory chemoreception in vivo (Richerson et al., 2001). These neurons represent a regulatory center for respiratory response under normoxic conditions and after traumatic brain injury. Neuronal acidosis occurs shortly after the ischemic insult and is accompanied by tachypnic respiratory response that originates in chemosensory neurons.

Several reports have indicated that chemosensory neurons

Received April 12, 2002; revised Aug. 12, 2002; accepted Aug. 12, 2002.

This work was supported in part by a grant-in-aid for scientific research from the Ministry of Education, Culture, Sports, Science, and Technology of Japan (N.S.) and the Strategic Funds of Sweden (I.D.). I.D. is a research fellow of the Boehringer Ingelheim Fonds. The DNA Data Bank of Japan/European Molecular Biology Laboratory/GenBank accession number for the nucleotide in this paper is AB075229.

Correspondence should be addressed to Noriaki Shimokawa, Molecular Signaling Group, Ludwig Institute for Cancer Research, Box 595, Husargatan 3, Uppsala, S-75124, Sweden. E-mail: simokawa@med.gunma-u.ac.jp.

Copyright (c) 2002 Society for Neuroscience 0270-6474/02/229160-06 $\$ 15.00 / 0$ adapt to hypercapnia or hypocapnia by triggering intracellular signaling pathways, leading to regulation of gene expression. For example, hypercapnic stimulation induces c-Fos/c-Jun expression in VMS neurons (Sato et al., 1992; Miura et al., 1998), whereas hypercapnia transiently decreased expression of brain-derived neurotrophic factor mRNA levels in the cingulate cortex (Uchino et al., 1997). More recently, we have shown that rat MafG homolog (Shimokawa et al., 2000) and Rhombex-29 (Shimokawa and Miura, 2000) genes are induced by hypercapnia. MafG is a nuclear transcription factor that forms heterodimers with c-Fos and recognizes DNA sequences that are quite similar to DNA binding sequences for Fos/Jun (Kataoka et al., 1994). The Rhombex-29 is a transmembrane (TM) protein, which is thought to be an important protein associated with $\mathrm{H}^{+}$sensitivity and adaptation to hypercapnia. Furthermore, applying a fluorescent $\mathrm{H}^{+}$indicator to cultured VMS neurons from neonatal rats, we detected significant $\mathrm{H}^{+}$ inflow into VMS neurons when the extracellular $\mathrm{pH}$ shifted to acidity (Sugama et al., 1997). These results suggested that proton transporters might be present in the plasma membrane of the $\mathrm{H}^{+}$-sensitive VMS neurons.

In the present study, we screened for differential genes that were expressed at a low level under normocapnic conditions but were induced significantly by low $\mathrm{pH}$ after hypercapnic stimulation. Here, we describe the identification of proton-associated sugar transporter-A (Past-A), a new class of sugar transporter that is induced by hypercapnia and is able to facilitate glucose uptake after low $\mathrm{pH}$. Because of its temporal and spatial expression in neuronal cells, Past-A may be involved in specific aspects of brain 
sugar $\mathrm{H}^{+}$metabolism that play critical roles in the adaptation to hypercapnia in the brain.

\section{MATERIALS AND METHODS}

Hypercapnic stimulation. Hypercapnic stimulation to rats was performed in the same manner as described previously (Miura et al., 1998). Briefly, 6- to 8-week-old male Wistar rats inhaled either air (normocapnic stimulation, $0.04 \% \mathrm{CO}_{2}$ ) or air containing $7 \% \mathrm{CO}_{2}$ (hypercapnic stimulation) for $5 \mathrm{~min}$ to stimulate the medullary chemoreceptor neurons. Thirty minutes after the cessation of inhalation procedures, the rats were decapitated. The superficial layer of the VMS was sliced $0.5 \mathrm{~mm}$ thick. The VMS slice covered a region $2 \mathrm{~mm}$ mediolateral to the midline and $1 \mathrm{~mm}$ rostral and $2 \mathrm{~mm}$ caudal to the anteroinferior cerebellar artery.

Differential display. Initially, $10 \mu \mathrm{g}$ of RNAs derived from the VMS slice after inhalation of either air or $7 \% \mathrm{CO}_{2}$ was exposed to RNase-free DNase I. Subsequently, $0.3 \mu \mathrm{g}$ of the treated RNAs was used as a template for reverse transcription. A $1 \mu \mathrm{l}$ volume of the reverse-transcribed cDNA was used for PCR. The nine anchored downstream oligo-dT primers were combined with 24 different upstream primers composed of 10 nucleotides. PCR products were loaded onto a $6 \%$ nondenaturing sequence gel and electrophoresed. Differentially amplified PCR fragments were visualized as mRNA fingerprints with a BAS-2000 Bio-Imaging Analyzer (Fujifilm, Tokyo, Japan). The target cDNA band was excised from the sequence gel, eluted, and reamplified with the same primer pair. The $5^{\prime}$ end of the target gene was amplified by the high-fidelity PCR-based $5^{\prime}$ - rapid amplification of cDNA ends with a primer pair consisting of the gene-specific primer 5'-CCTGCAGCAGACCACCTTCAAGTCC-3' and the adapter primer 5'-CCATCCTAATACGACTCACTATAGGGC-3'. The cDNA sequence of Past-A and the phylogenic tree of different sequence alignments were analyzed using the BLAST algorithms (Altschul et al., 1990) and by the Clustal W program (Thompson et al., 1994), respectively

Northern blotting and immunohistochemistry. To detect RNAs, Northern hybridization was used in the same manner as described previously (Shimokawa and Miura, 2000). In the immunohistochemical experiments with Past-A, 90 min after the cessation of inhalation of gases, normocapniaor hypercapnia-treated rats were anesthetized with pentobarbital sodium $(50 \mathrm{mg} / \mathrm{kg}$ body weight, i.p.) and perfused transcardially with heparinized saline, followed by $0.5 \%$ glutaraldehyde and $4 \%$ paraformaldehyde. After fixation of the brains, the medulla oblongata and pons were cut at $40 \mu \mathrm{m}$ in frontal series, and sections were incubated with rabbit anti-Past-A polyclonal antiserum raised against an N-terminal peptide (IPPAGSTPPGEALIPSC). Subsequently, the sections were incubated with biotinylated anti-rabbit IgG antiserum (Vector Laboratories, Burlingame, CA). After that, the sections were processed with avidin-biotin peroxidase complex and treated with diaminobenzidine solution containing $0.003 \% \mathrm{H}_{2} \mathrm{O}_{2}$.

Biochemical analyses. Complementary DNAs for Past-A and glucose transporter 4 (GLUT4) were subcloned into pcDNA3 (Invitrogen, Carlsbad, CA). COS-7 cells were transfected with the indicated vectors by using the lipid-based transfection reagent FuGENE 6 (Roche, Basel, Switzerland). For membrane separations, transfected COS-7 cells were harvested and fractionated as described previously (Simpson et al., 1983). Proteins from membrane fraction $(10 \mu \mathrm{g})$ were analyzed by Western blotting using anti-Past-A or GLUT4 (Santa Cruz Biotechnology, Santa Cruz, CA) polyclonal antibody. Sugar uptake was measured as described previously (Chen et al., 1997) with minor modifications. In brief, $64 \mathrm{hr}$ after transfection, cells were washed with KRPH buffer (in mM: 20 HEPES, pH 7.4, $5 \mathrm{Na}_{2} \mathrm{HPO}_{4}, 1.25 \mathrm{MgSO}_{4}, 1.25 \mathrm{CaCl}_{2}, 136 \mathrm{NaCl}$, and 4.7 $\mathrm{KCl}$ ). Sugar uptake was determined by incubation with $\mathrm{KRPH}$ buffer containing one of the following: $50 \mu \mathrm{M}{ }^{3} \mathrm{H}$-labeled glucose, 3-Omethylglucose, galactose, fructose, or sucrose $(0.5 \mu \mathrm{Ci}$; Amersham Biosciences, Piscataway, NJ) and either untreated, with added protonophore, or with changed $\mathrm{pH}$ of the buffer as described in the figure legends. The cells were washed with KRPH buffer and solubilized in 1\% Triton X-100. The radioactivity incorporated into the cells was measured with a liquid scintillation counter.

\section{RESULTS}

\section{Isolation and analysis of Past-A gene}

Differential display technique was used to identify the genes responsible for adaptation to hypercapnia by comparing profiles of mRNAs in the VMS after inhalation of $7 \% \mathrm{CO}_{2}$ and air $\left(0.04 \% \mathrm{CO}_{2}\right)$. More than 11,500 PCR products were generated, and $14(0.12 \%)$ of the observed bands exhibited profiles of high- expression genes by hypercapnic stimulation. We focused on a novel clone that was particularly interesting because of its unique expression profile and primary structure resembling sugar transporters. We named it Past- $A$.

The cDNA of Past-A contains an open reading frame encoding a sequence of 751 amino acids (Fig. $1 A$ ), and the relative molecular weight of the residues was calculated as $\sim 82 \mathrm{kDa}$. Analysis of the predicted amino acid sequence suggested the presence of 12 putative membrane-spanning helices with a long cytoplasmic loop between TM6 and TM7 and with intracellular orientation of N and $\mathrm{C}$ termini (Fig. $1 B$ ). Several motifs that have been shown to be critical for the sugar transport function are conserved. These include the sequence motifs RXGRR (in which $\mathrm{X}$ is any amino acid: RFGRR in Past-A) between TM2 and TM3, RG between TM4 and TM5, PESP motif (PERP in Past-A) after helix 6, and QLS motif (WLS in Past-A) in TM helix 7. Moreover, the region between TM2 and TM3 has a completely conserved sucrose- $\mathrm{H}^{+}$ transport motif; it is found in all known sucrose $-\mathrm{H}^{+}$transporters in plants. In addition, motifs corresponding to three proline-rich motifs, one in the $\mathrm{N}$ terminus and two after helix 6 , and leucinezipper structure in helix 9 are present in Past-A. Figure $1 C$ shows a phylogenic tree of the sequence relatedness of Past-A and the closest transporter molecules. Past-A shows the highest similarity to membrane-associated transporter protein B of Oryzias latipes (medaka) and its human and mouse homologs, antigen isolated from immunoselected melanoma (AIM)-1s. These three molecules contain an intracellular loop between TM2 and TM3, which shows an average of $67.9 \%$ identity with the corresponding region in the Past-A sequence. The region from TM2 to TM3 contains a sucrose $-\mathrm{H}^{+}$transport motif, and the motif of Past-A shows $86.4 \%$ identity with spinach sucrose $-\mathrm{H}^{+}$transport motif (Riesmeier et al., 1992). The phylogenic tree also shows that other closely related proteins include the rat GLUT family.

\section{Tissue distribution and expression of Past-A}

To examine the tissue specificity of Past- $A$ expression, adult rat RNAs extracted from various tissues were analyzed by Northern blotting using rat Past-A cDNA as a probe (Fig. $2 A$ ). Past-A mRNA is expressed highly in the VMS and moderately in the cerebral cortex and cerebellum. To examine Past- $A$ expression in the developing brain, RNA from embryonic day 20 (E20), postnatal day 2 (P2), P50, and P300 rat brain was also analyzed (Fig. $2 B$ ). Expression of Past- $A$ transcript had begun by at least E20, and, as the embryos grew into adult rats, constant expression of Past-A mRNA was found in the brain.

To confirm whether hypercapnic stimulation induces expression of Past-A, we performed Northern blot analysis of mRNA isolated from the VMS of rats under $\mathrm{CO}_{2}$ or air inhalation. Hypercapnic stimulation induced approximately threefold expression of Past-A mRNA in the VMS compared with the results after air inhalation (Fig. $3 A$ ). Figure $3 B$ shows that the density of Past-A mRNA in the VMS was $151 \pm 19.6 \mathrm{U}$ after air breathing (control) and increased significantly to $472 \pm 46.1 \mathrm{U}$ after $7 \%$ $\mathrm{CO}_{2}$ inhalation.

The localization of Past-A protein in the brain was examined immunohistochemically using a polyclonal antibody against the Past-A peptide sequence. Past-A-immunoreactive neurons were found primarily in the VMS (Fig. 3C). We could not detect any Past-A immunoreactivity in other areas or nuclei in the brain stem. After exposure to hypercapnic stimulation, the number of Past-A-immunoreactive neural cells in the VMS was increased 
A

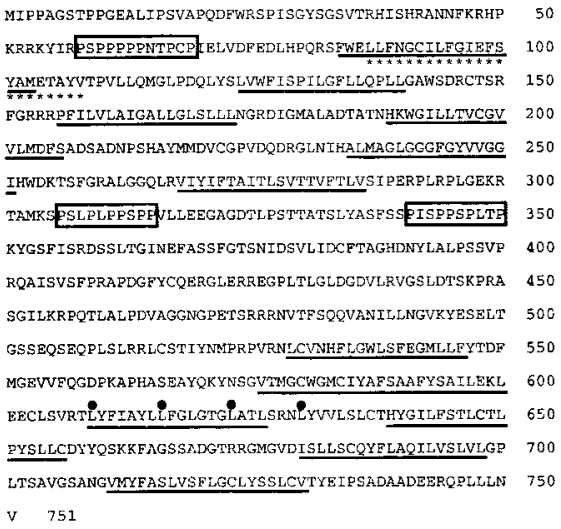

C

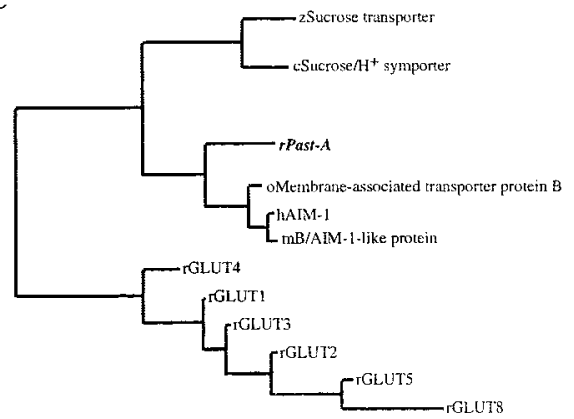

Figure 1. Structural analysis of Past-A. A, Amino acid sequences of rat Past-A. The approximate positions of the transmembrane domains are underlined. Three proline-rich regions are boxed. The leucine repeats are marked with filled circles. The sucrose $-\mathrm{H}^{+}$transport motif is indicated with asterisks. Numbers to the right show amino acid positions. $B$, Putative membrane topology of Past-A. Three prolinerich regions are boxed. The leucine repeats are marked with dark background. The sucrose $-\mathrm{H}^{+}$transport motif is indicated with asterisks. Numbers show amino acid positions. $C$, Phylogenic tree of a multiple alignment the Past-A with other transporters. $c$, Carrot; $h$, human; $m$, mouse; o, Oryzias latipes (medaka); $r$, rat; $z$, zea mays (maize).

\section{B}

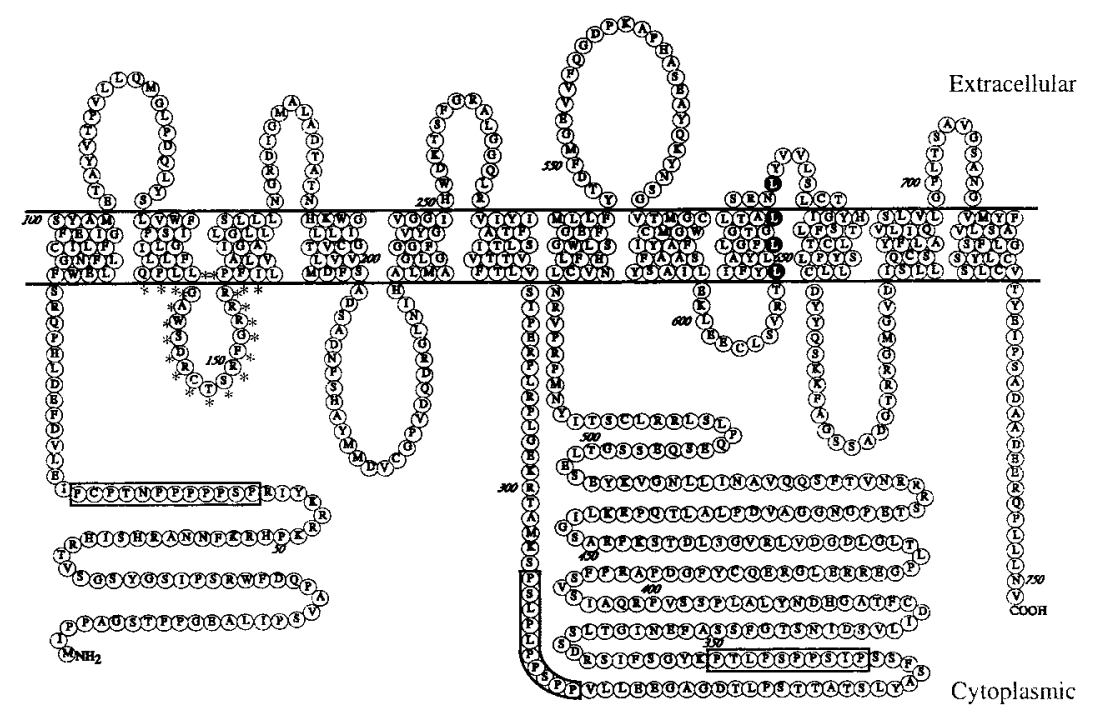

approximately fourfold compared with the results after air inhalation (Fig. 3D).

\section{Glucose transport activity of Past-A}

To determine whether Past-A is a functional sugar transporter, we measured transport activity of Past-A in the presence of radiolabeled sugar molecules. Expression of Past-A in COS-7 cells led to a 4.1-fold increase in D-glucose transport activity compared with mock-transfected cells (Fig. 4A). Uptake could be blocked by cytochalasin B, a specific inhibitor of the mammalian glucose transporter isoforms (data not shown). Transfection with GLUT4 cDNA as a positive control produced a 6.7-fold increase in glucose uptake. Expression of Past-A also increased uptake of galactose but not sucrose and fructose in the same cells. The expression of Past-A $(82 \mathrm{kDa})$ at the plasma membrane was confirmed by immunoblotting with an antibody against the N-terminal peptide of Past-A (Fig. 4B). Western blotting with anti-GLUT4 antibody showed a band of $45 \mathrm{kDa}$, as expected.

To examine the glucose transport activity of Past-A after changes in extracellular $\mathrm{pH}$, either protonophore, an agent that collapses the $\mathrm{pH}$ gradient across the cell membrane, was used or cells were incubated in culture medium with an excess of $\mathrm{H}^{+}$, thus inducing lower $\mathrm{pH}$ in the medium. Glucose uptake activity of Past-A was decreased significantly when the concentration of the protonophore carbonyl cyanide $m$-chlorophenylhydrazone (CCCP) was increased gradually from 1 to $25 \mu \mathrm{M}$ (Fig. 4C), suggesting that an intact proton gradient was critical for the ability of Past-A to mediate glucose uptake. Figure $4 D$ shows a correlation between glucose uptake activity of Past-A and extracellular $\mathrm{pH}$. A significant increase in glucose uptake was detected at lower $\mathrm{pH}$ values ( $\mathrm{pH} 7.0$ and 6.5) as early as 1 min after incubation with acidic media. Incubation in culture medium at an extracellular $\mathrm{pH}$ of 7.0 and 6.5 produced a 2.8- and 3.8-fold increase in glucose uptake activity, respectively, compared with the value at an extracellular $\mathrm{pH}$ of 7.5 after 10 min incubation. This indicates that sugar transport activity of Past-A is dependent on the membrane $\mathrm{pH}$ gradient. The uptake of D-glucose reflects the rate of transmembrane transport. Nonetheless, the effect of low $\mathrm{pH}$ on glucose uptake may have been a result of modulation of hexokinase activity, the enzyme that phosphorylates 2-deoxyD-glucose to 2-deoxy-D-glucose 6-phosphate. Therefore, we also tested whether incubation in low $\mathrm{pH}(\mathrm{pH} 7.0$ and 6.5) affects glucose transport using 3-O-methylglucose (a nonphosphorylatable analog of glucose). After $10 \mathrm{~min}$ of incubation in low $\mathrm{pH}$, 3-O-methylglucose transport was increased 2.2-fold at a $\mathrm{pH}$ of 7.0 and 3.1-fold at a $\mathrm{pH}$ of 6.5 relative to the uptake in cells incubated at a $\mathrm{pH}$ of 7.5 (data not shown).

\section{DISCUSSION}

Differential display is an established technique that allows for the microanalysis of transcriptional changes occurring in a given cell or tissue (Liang and Pardee, 1992). This method has been verified to be effective for identifying novel and differentially expressed 
A
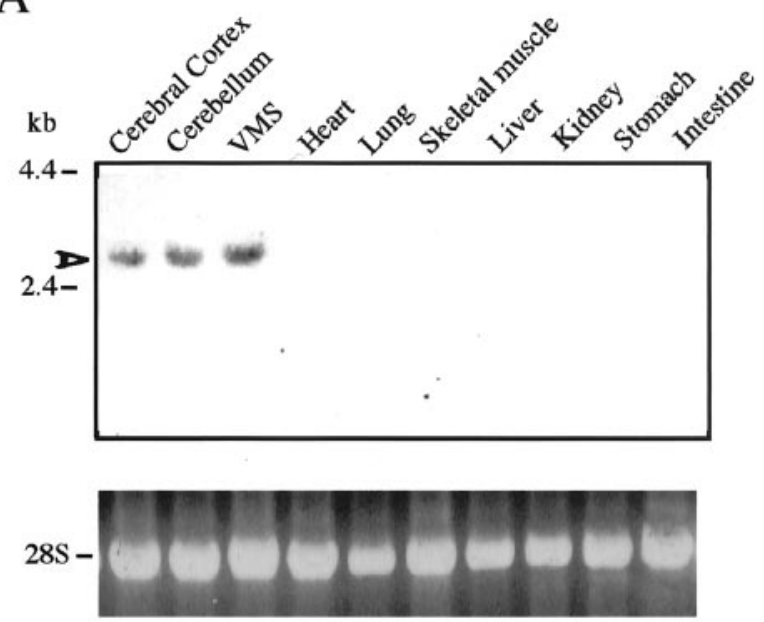

B

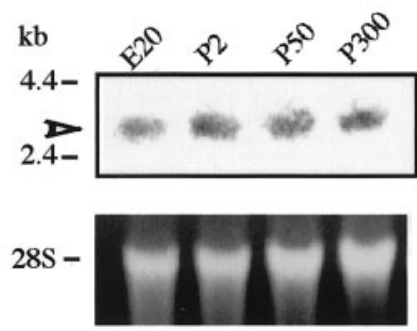

Figure 2. Tissue distribution and ontogenic changes of Past-A mRNA. Total RNAs $(20 \mu \mathrm{g})$ extracted from various tissues $(A)$ or from brains at different developmental stages (E20 to P300) $(B)$ were analyzed by Northern blot. Arrowheads mark hybridizing bands corresponding to mRNA encoding rat Past-A. The positions of standard molecular masses (in kilobases) are indicated at left. Ribosomal RNA (28S) stained with ethidium bromide is shown as a control.

genes at various physiological aspects in CNS neural cells, such as RNA-binding peptide RA310 induced by hypoxia-reoxygenation (Matsuo et al., 1995), seizure-induced synaptotagmin gene syt $X$ (Babity et al., 1997), zinc transporter ZnT-1 cloned after forebrain ischemia (Tsuda et al., 1997), and membrane glycoprotein Pal expressed specifically in photoreceptor cells of the retina (Gomi et al., 2000). Here, we describe identification and characterization of Past-A, a novel class of mammalian sugar transporter, highly enriched in brain, that couples $\mathrm{H}^{+}$gradients with glucose uptake. Past-A is expressed specifically in the VMS neuronal cells and is induced by hypercapnia. Primary structure analysis indicated that the Past-A protein belongs to the sugar porter family in the major facilitator superfamily (Pao et al., 1998). These proteins have 12 transmembrane-spanning helices. Substrates transported by the sugar porter family members include pentoses (arabinose and xylose), hexoses (glucose, fructose, and galactose), disaccharides (sucrose, maltose, and lactose), inositols, quinate, and cations. Some evidence indicates that Past-A is a functional glucose transporter. In transfected cells, Past-A mediates glucose uptake (Fig. 4). Past-A also has the same membrane topology as the glucose transporters, with 12 membranespanning helices having a long cytoplasmic loop between TM6 and TM7, and several motifs described to be important for glucose transport activity are conserved, including the RXGRR motif and PESP motif after helix 6 (Fig. $1 A$ ). In addition to glucose, Past-A was shown to facilitate uptake of galactose but not

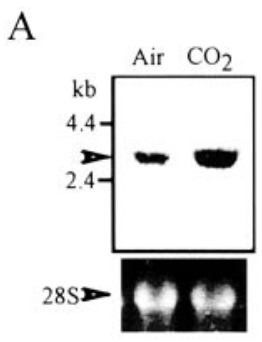

$\mathrm{B}$

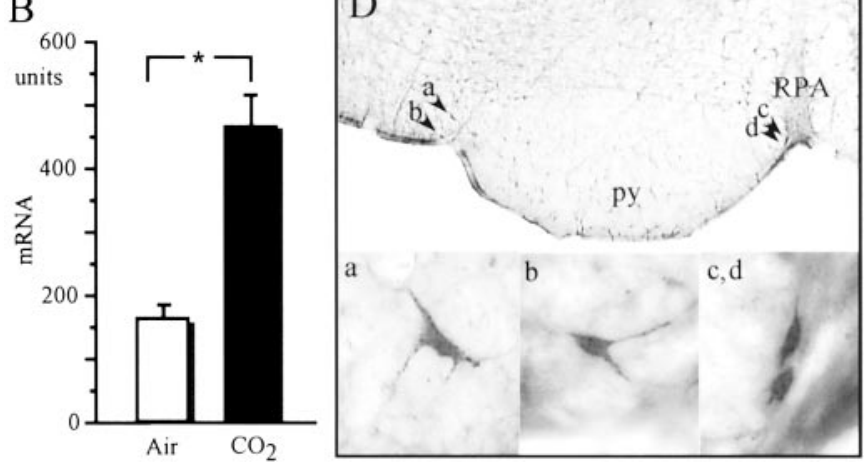

Figure 3. Induction of Past-A by hypercapnic stimulation in the medulla oblongata. $A$, Analysis of differential expression of Past- $A$ by hypercapnic stimulation by Northern blotting. Representative example of autoradiogram sampled from four experiments. Arrowhead shows hybridizing band corresponding to mRNA encoding rat Past-A. Positions of standard molecular masses (in kilobases) are indicated at left. Ribosomal RNA (28S) stained with ethidium bromide is shown as a control. $B$, Histograms of density units of the expression of Past-A mRNA after air or $\mathrm{CO}_{2}$ inhalation. The content of Past-A mRNA was measured densitometrically on the autoradiogram, and the results are expressed as means \pm SEM of arbitrary units of density $(n=4)$. The significance of differences in the density of Past-A mRNA was evaluated by the Mann-Whitney $U$ test, with $* p<0.05$ considered indicative of a statistically significant difference. $C$, Photomicrographs of Past-A-immunoreactive neural cells in the VMS after inhalation of air. Right, Magnified subregion indicated by arrow at left. D, Photomicrographs of Past-A-immunoreactive neuronal cells in the VMS after inhalation of air containing $7 \% \mathrm{CO}_{2}$ for $5 \mathrm{~min}$. Bottom, Magnified subregions indicated by arrows at top. py, Pyramidal tract; $R P A$, nucleus raphe pallidus. Scale bars: panoramic picture, $200 \mu \mathrm{m}$; magnified picture, $20 \mu \mathrm{m}$.

of fructose or sucrose (Fig. $4 A$ ). It is also interesting to mention that Past-A is most closely related to a novel transporter-like protein, AIM-1 (Fig. 1C). AIM-1 was suggested to transport substances including galactose or certain saccharides that are required for melanin biosynthesis in the medaka (a small, freshwater teleost) (Fukamachi et al., 2001). Glucose transport mediated via Past-A was facilitated rapidly in the presence of low $\mathrm{pH}$, whereas it was inhibited in the presence of protonophore, suggesting that its glucose transporter activity is controlled by the $\mathrm{pH}$ gradient. Protonophores such as CCCP are agents that collapse the $\mathrm{pH}$ gradient across the cell membrane. They also affect the $\mathrm{H}^{+}$gradient across the inner mitochondrial membrane and as a result disturb electron transport through the respiratory chain and ATP generation. Because it is possible that CCCP affects activity of symporter or hexokinase through change of the energy state (ATP generation) in mitochondria, additional work is necessary with regard to the glucose transport activity in a $\mathrm{pH}$ dependent manner of Past-A. Because Past-A has a completely conserved sucrose $-\mathrm{H}^{+}$transport motif of plants, it is possible that Past-A is a sugar- $\mathrm{H}^{+}$symporter. However, additional work will 

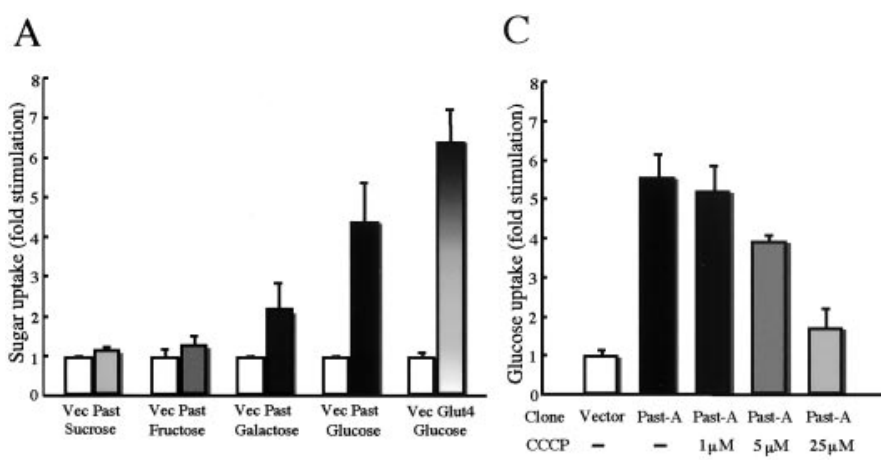

B
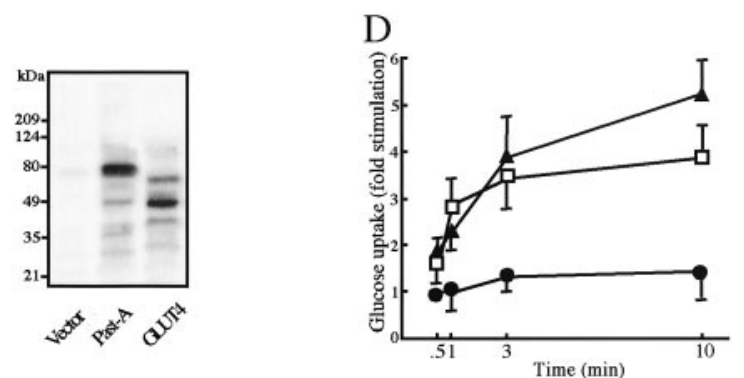

Figure 4. Characterization of sugar transport activity of Past-A. A, COS-7 cells were transfected with blank vector $(V e c)$, Past-A (Past), or rat GLUT4 cDNA. Sixty-four hours after transfection, transfected cells were incubated with one of radiolabeled sugars and solubilized as described in Materials and Methods. Results are averages of four individual experiments, with SEM indicated by error bars. B, Western blot of the membrane fractions. Transporter proteins Past-A and GLUT4 were detected with each antiserum. The positions of standard molecular masses (in kilodaltons) are indicated at left. $C$, Inhibition of glucose uptake of COS-7 transfected with Past-A cDNA by the protonophore CCCP. Glucose uptake data are expressed as fold stimulation relative to the extent of uptake observed with COS-7 transfected with blank vector not exposed to CCCP. Results are averages of four individual experiments, with SEM indicated by error bars. $D$, COS-7 transfected cells with Past-A cDNA were incubated at different $\mathrm{pH}$ values for glucose uptake. Glucose uptake data are expressed as fold stimulation relative to the extent of uptake observed with COS-7 transfected with Past-A cDNA at a pH of 7.5. Results are averages of four individual experiments, with SEM indicated by error bars. $(\boldsymbol{\Delta}), \mathrm{pH} 6.5 ;(\square), \mathrm{pH} 7.0 ;(\bullet), \mathrm{pH} 7.5$.

be needed to clarify whether Past-A mediates the influx of $\mathrm{H}^{+}$ into neuronal cells together with glucose uptake.

There are two unique features in the Past-A molecule that may be of relevance for additional functions of Past-A inside the cell. One is the presence of proline-rich regions in the long intracellular loop present between TM6 and TM7 and in the N terminus of Past-A that are not found in other sugar transporters, such as mammalian GLUTs, or in the related sucrose $-\mathrm{H}^{+}$transporters. A polyproline motif (core sequence, PXXP, in which $\mathrm{X}$ denotes any amino acid) is thought to be involved in specific proteinprotein interactions (Kay et al., 2000). Past-A may therefore serve as a binding partner for Src homology 3-domain-containing proteins independently of glucose transporter function. Recent study has indicated that glucose via GLUT1 transporter stimulates the tyrosine kinase-dependent cascade, leading to activation of the mitogen-activated protein (MAP) kinase pathway (Bandyopadhyay et al., 2000). Few studies have shown that acidification of extracellular $\mathrm{pH}$ by hypercapnia triggers intracellular signal transduction, including induction of c-Fos mRNA expression through $\mathrm{PKC}, \mathrm{Ca}^{2+} /$ calmodulin, and MAP kinase pathways (Kuo et al., 1998; Shimokawa et al., 1998). The other feature of Past-A is the leucine zipper structure in TM9. The leucine zipper con- sists of a periodic repetition of leucine residues and was implicated in the facilitation of protein dimerization (Landschulz et al., 1988). One possibility is that leucine zipper structures in the transmembrane helix may be involved in the formation of transmembrane topology by interaction with other transmembrane proteins.

Neurons of the medulla oblongata have been show to act as a regulatory center for respiratory response as well as the glucosesensing system in the brain (Oomura et al., 1974; Ritter et al., 1992; Yettefti et al., 1995). The area postrema and the nucleus tractus solitarius in the medulla oblongata possess the glucose sensors related to feeding and reproduction (Ritter et al., 1992). Glucose-sensing neurons respond to hyperglycemia or hypoglycemia by increasing or decreasing their firing rates (Oomura et al., 1974; Yettefti et al., 1995). The role of chemosensitive and glucose-sensing neurons is critical in both physiological and pathophysiological conditions. Pathological changes that accompany brain trauma often include brain acidosis, hypercapnia, hypoglycemia, and ischemic hypoxia. Expression of Past-A is increased significantly in neurons of the VMS in response to acidification of extracellular $\mathrm{pH}$ by hypercapnia. Our results also indicate a functional role for Past-A in controlling glucose uptake along the $\mathrm{pH}$ gradient, suggesting that hypercapnia may stimulate uptake of glucose, a main energy source, into acidosis-stressed neurons of the VMS. Future functional analysis of Past-A may provide new insights into the biochemical regulation of glucosesensing mechanisms in the brain, in addition to adaptation to hypercapnia.

\section{REFERENCES}

Altschul SF, Gish W, Miller W, Myers EW, Lipman DJ (1990) Basic local alignment search tool. J Mol Biol 215:403-410.

Babity JM, Armstrong JN, Plumier JC, Currie RW, Robertson HA (1997) A novel seizure-induced synaptotagmin gene identified by differential display. Proc Natl Acad Sci USA 94:2638-2641.

Bandyopadhyay G, Sajan MP, Kanoh Y, Standaert ML, Burke TR, Quon MJ, Reed BC, Dikic I, Noel LE, Newgard CB, Farese R (2000) Glucose activates mitogen-activated protein kinase (extracellular signal-regulated kinase) through proline-rich tyrosine kinase-2 and the Glut1 glucose transporter. J Biol Chem 275:40817-40826.

Chen D, Elmendorf JS, Olson AL, Li X, Earp HS, Pessin JE (1997) Osmotic shock stimulates GLUT4 translocation in 3T3L1 adipocytes by a novel tyrosine kinase pathway. J Biol Chem 272:27401-27410.

Fukamachi S, Shimada A, Shima A (2001) Mutations in the gene encoding $\mathrm{B}$, a novel transporter protein, reduce melanin content in medaka. Nat Genet 28:381-385.

Fukuda Y, Honda Y (1975) pH-sensitive cells at ventro-lateral surface of rat medulla oblongata. Nature 256:317-318.

Gomi F, Imaizumi K, Yoneda T, Taniguchi M, Mori Y, Miyoshi K, Hitomi J, Fujikado T, Tano Y, Tohyama M (2000) Molecular cloning of a novel membrane glycoprotein, Pal, specifically expressed in photoreceptor cells of the retina and containing leucine-rich repeat. J Neurosci 20:3206-3213.

Kataoka K, Noda M, Nishizawa N (1994) Maf nuclear oncoprotein recognizes sequences related to an AP-1 site and forms heterodimers with both Fos and Jun. Mol Cell Biol 14:700-712.

Kay BK, Williamson MP, Sudol M (2000) The importance of being proline: the interaction of proline-rich motifs in signaling proteins with their cognate domains. FASEB J 14:231-241.

Kuo NT, Agani FH, Haxhiu MA, Chang CH (1998) A possible role for protein kinase $\mathrm{C}$ in $\mathrm{CO}_{2} / \mathrm{H}^{+}$-induced c-fos mRNA expression in $\mathrm{PC} 12$ cells. Respir Physiol 111:127-135.

Landschulz WH, Johnson PF, McKnight SL (1988) The leucine zipper: a hypothetical structure common to a new class of DNA binding proteins. Science 240:1759-1764.

Liang P, Pardee AB (1992) Differential display of eukaryotic messenger RNA by means of the polymerase chain reaction. Science 257:967-971.

Loeschcke HH (1982) Central chemosensitivity and the reaction theory. J Physiol (Lond) 332:1-24.

Matsuo N, Ogawa S, Imai Y, Takagi T, Tohyama M, Stern D, Wanaka A (1995) Cloning of a novel RNA binding polypeptide (RA301) induced by hypoxia/reoxygenation. J Biol Chem 270:28216-28222.

Miura M, Okada J, Kanazawa M (1998) Topology and immunohisto- 
chemistry of proton-sensitive neurons in the ventral medullary surface of rats. Brain Res 780:34-45.

Oomura Y, Ooyama H, Sugimori M, Nakamura T, Yamada Y (1974) Glucose inhibition of the glucose-sensitive neurone in the rat lateral hypothalamus. Nature 247:284-286.

Pao SS, Paulsen IT, Saier MH (1998) Major facilitator superfamily. Microbiol Mol Biol Rev 62:1-34.

Richerson GB, Wang W, Tiwari J, Bradley SR (2001) Chemosensitivity of serotonergic neurons in the rostral ventral medulla. Respir Physiol 129:175-189.

Riesmeier JW, Willmitzer L, Frommer WB (1992) Isolation and characterization of a sucrose carrier cDNA from spinach by functional expression in yeast. EMBO J 11:4705-4713.

Ritter S, Calingasan NY, Hutton B, Dinh TT (1992) Cooperation of vagal and central neural systems in monitoring metabolic events controlling feeding behavior. In: Neuroanatomy and physiology of abdominal vagal afferents (Ritter S, Ritter RC, Barnes CD, eds), pp 249-277. Boca Raton, FL: CRC.

Sato M, Severinghaus JW, Basbaum AI (1992) Medullary $\mathrm{CO}_{2}$ chemoreceptor neuron identification by c-fos immunocytochemistry. J Appl Physiol 73:96-100.

Schlaefke ME, See WR, Loeschcke HH (1970) Ventilatory response to alterations of $\mathrm{H}^{+}$ion concentration in small area of the ventral medullary surface. Respir Physiol 10:198-212.

Shimokawa N, Miura M (2000) Rhombex-29, a novel gene of the PLP/ DM20-M6 family cloned from rat medulla oblongata by differential display. J Neurosci Res 62:1-8.

Shimokawa N, Sugama S, Miura M (1998) Extracellular $\mathrm{H}^{+}$stimulates the expression of $c$-fos/c-jun mRNA through $\mathrm{Ca}^{2+} /$ calmodulin in PC12 cells. Cell Signal 10:499-503.

Shimokawa N, Okada J, Miura M (2000) Cloning of MafG homologue from the rat brain by differential display and its expression after hypercapnic stimulation. Mol Cell Biochem 203:135-141.

Simpson IA, Yver DR, Hissin PJ, Wardzala LJ, Karnieli E, Salans LB, Cushman SW (1983) Insulin-stimulated translocation of glucose transporters in the isolated rat adipose cells: characterization of subcellular fractions. Biochim Biophys Acta 763:393-407.

Sugama S, Shimokawa N, Okada J, Miura M (1997) In vitro study of $\mathrm{H}^{+}$-sensitive neurons in the ventral medullary surface of neonate rats. Brain Res 777:95-102.

Thompson JD, Higgins DG, Gibson TJ (1994) CLUSTAL W: improving the sensitivity of progressive multiple sequence alignment through sequence weighting, position-specific gap penalties and weight matrix choice. Nucleic Acids Res 22:4673-4680.

Tsuda M, Imaizumi K, Katayama T, Kitagawa K, Wanaka A, Tohyama M, Takagi T (1997) Expression of zinc transporter gene, ZnT-1, is induced after transient forebrain ischemia in the gerbil. J Neurosci 17:6678-6684.

Uchino H, Lindvall O, Siesjo BK, Kokaia Z (1997) Hyperglycemia and hypercapnia suppress BDNF gene expression in vulnerable regions after transient forebrain ischemia in the rat. J Cereb Blood Flow Metab 17:1303-1308.

Yettefti K, Orsini JC, El Ouazzani T, Himmi T, Boyer A, Perrin J (1995) Sensitivity of nucleus tractus solitarius neurons to induced moderate hyperglycemia, with special reference to catecholaminergic regions. J Auton Nerv Syst 51:191-197. 